\title{
Investigation of Simultaneous Photons and Electrons Beam by Monte Carlo Code
}

\author{
Navid Khaledi, ${ }^{1}$ Azim Arbabi, ${ }^{2}$ Dariush Sardari,', Mohammad Mohammadi, ${ }^{3}$ and \\ Ahmad Ameri ${ }^{2}$ \\ ${ }^{1}$ Department of Medical Radiation, Science and Research Branch, Islamic Azad University, Tehran, IR Iran \\ 2Department of Radiotherapy, Imam Hossein Hospital, Shahid Beheshti University of Medical Sciences, Tehran, IR Iran \\ ${ }^{3}$ Department of Medical Physics, Hamedan University of Medical Sciences, Hamedan, IR Iran \\ *Corresponding author: Dariush Sardari, Department of Medical Radiation, Science and Research Branch, Islamic Azad University, Tehran, IR Iran. \\ E-mail: dsardari94@gmail.com \\ Received: May 15, 2015; Accepted: May 26, 2015
}

\begin{abstract}
Background: Depending on the location and depth of tumor, the electron or photon beams might be used for treatment. Electron beam have some advantages over photon beam for treatment of shallow tumors to spare the normal tissues beyond of the tumor. In the other hand, the photon beam are used for deep targets treatment. Both of these beams have some limitations, for example, the dependency of penumbra with depth, and the lack of lateral equilibrium for small electron beam fields.

Objectives: In this study, improvement of the penumbra and Dmax changes will be investigated. Also the effects of cut-outs on the beam parameters prepared as well.

Patients and Methods: In first, we simulated the conventional head configuration of Varian 2300 for $16 \mathrm{MeV}$ electron, and the results approved by benchmarking the percent depth dose (PDD) and profile of the simulation and measurement. In the next step, a perforated Lead $(\mathrm{Pb})$ sheet with $1 \mathrm{~mm}$ thickness placed at the top of the applicator holder tray. This layer producing bremsstrahlung $\mathrm{x}$-ray and a part of the electrons passing through the holes, in result, we have a simultaneous mixed electron and photon beam. For making the irradiation field uniform, a layer of steel placed after the Pb layer. The simulation was performed for $10 \times 10$, and $4 \times 4 \mathrm{~cm}^{2}$ field size.

Results: The measured R50 and RP for $10 \times 10 \mathrm{~cm} 2$ field were 6.5 and $7.8 \mathrm{~cm}$, respectively. The photon percentage for $1 \mathrm{~mm}$ thickness with 0.2 , 0.3 , and $0.5 \mathrm{~cm}$ holes diameter Lead layer target was about $33 \%, 32 \%$, and $28 \%$ and for $2 \mathrm{~mm}$ targets punched with $0.2,0.3$, and $0.5 \mathrm{~cm}$ holes, the $\mathrm{x}$-ray percentages were $43 \%, 41 \%$, and $35 \%$.

Conclusions: This study showed the advantages of mixing the electron and photon beam by reduction of pure electron's penumbra dependency with the depth, especially for small fields, also decreasing of dramatic changes of PDD curve with irradiation field size.
\end{abstract}

Keywords: Monte Carlo Methods; Photons; Electrons

\section{Background}

Electron beam is widely used for treatment of non-deep lesions, because of its most important advantage over the photon beam: lesser dose delivery to normal tissues beyond of the tumor, due to its steep dose gradient relative to the depth. This capability leads to employ it in radiation therapy of nasal, breast, and parotid regions $(1,2)$. On the other side, its usage is limited by the dependency of penumbra to the depth and influence of dose parameters of electron beam from the field size changing (3-8). The issue of penumbra increment by depth is enhanced in some investigations by adding a narrow photon beam to the edge of the electron beam field (5) or mixing a low weight photon to electron beam field $(4,9,10)$. To our knowledge, no study on small fields for mixed electron and photon beam has been reported. In additional, none of these studies carried out for producing the simultaneous mixed electron and photon beam, they mixed the beams separately with conventional configuration of the linac machine.

\section{Objectives}

In this study, improvement of the penumbra and Dmax changes will be investigated. Also, the effects of cut-outs on the beam parameters prepared as well.

\section{Materials and Methods}

\subsection{Measurements}

For tuning of the MC code, the PDD and profile curve of $16 \mathrm{MeV}$ electron beam of Varian 2300/CD linac was measured. The profile measurements was obtained at the $1,3.5$, and $7 \mathrm{~cm}$ depths. The PTW parallel plane Markus camber type 34045 with $0.02 \mathrm{~cm}^{3}$ sensitive volume was used for percentage depth dose (PDD) and profile measurements of $10 \times 10 \mathrm{~cm}^{2}$ applicator in PTW 3D water phantom MP3 at $100 \mathrm{~cm}$ source to surface distance (SSD) condition. The air gap between bottom

Copyright (C)2015, Iranian Society of Clinical Oncology. This is an open-access article distributed under the terms of the Creative Commons Attribution-NonCommercial 4.0 International License (http://creativecommons.org/licenses/by-nc/4.0/) which permits copy and redistribute the material just in noncommercial usages, provided the original work is properly cited. 
side of applicator and the water phantom surface was 5 $\mathrm{cm}$. The PDD was obtained from percentage depth ionization (PDI) conversion according to the IAEA TRS 398 protocol (11).

\subsection{Monte Carlo Simulation}

By employing the MCNPX version 2.6 (12) running in a PC with core i5 Intel processor and 4 GB RAM, the simulations has performed.

The primary and secondary scattering foil of Varian $2300 / C D$ linac head was simulated. The primary scattering foil had a $0.5 \mathrm{~mm}$ thickness Aluminum foil, and the secondary scattering foil was a Tantalum cone with base radius of $20 \mathrm{~mm}$ and $5 \mathrm{~mm}$ height $(13,14)$. The source had $1.15^{\circ}$ angular distribution. The collimator jaws were composed of Tungsten and the applicator scrapers made of zinc alloy (15) and the standard cutout of applicator bottom was of Cerrobend alloy (52.5\% $\mathrm{Bi}, 32 \% \mathrm{~Pb}, 15.5 \% \mathrm{Sn}$ ). The simulated water phantom was a $50 \times 50 \times 50 \mathrm{~cm}^{3}$ of $\mathrm{H}_{2} \mathrm{O}$. For the PDD calculation the central axis in water phantom was divided into $0.2 \times$ $0.2 \times 0.2 \mathrm{~cm}^{3}$ cubes (voxels). For dose profile calculation the depths of $1,3.5$, and $7 \mathrm{~cm}$ were divided to the same voxels. By employing Mesh Tally type 3 the energy deposition in each voxels were calculated. The mesh tally type 3 scores the energy deposition from all particles. The tally values of each voxel constitutes the points of PDD and dose profile curves.

In the next step, for producing the simultaneous mixed electron and photon beam, the $\mathrm{Pb}$ sheet (perforated target) with the thickness of 1 and $2 \mathrm{~mm}$ punched with $0.2,0.3$, and $0.5 \mathrm{~cm}$ diameter holes. The center-to-center distance of these holes were $0.9 \mathrm{~cm}$. These cylindrical holes allows to the electrons to passing through them, and on the other hand, the striking of electrons on the Lead sheet produces the bremsstrahlung photon beam. This layer was placed at $39 \mathrm{~cm}$ from the phantom surface, above the applicator holder tray (first scraper of applicator). The reason for choosing the $\mathrm{Pb}$ for $\mathrm{x}$-ray production are: its high atomic number $(Z=82)$, low price and easy molding. According to provided documents by Siemens and Varian linac manufactures, the linac target is made of Gold $(Z=79)$ or Tungsten $(Z=74)$.

At the bottom of the Lead layer, at the distance of 37 $\mathrm{cm}$ from the phantom surface, the scattering component is placed. This component is used for scattering of the passed electrons from the Lead layer for uniform the electron beam distribution in the radiation field. It is composed of two layers: 1 -a wide steel layer which covers the whole of passed electrons through the Pb layer, act as scattering layer for all electrons, $2-\mathrm{a} 3 \times 3 \mathrm{~cm}^{2}$ steel layer at the top of the first layer for reducing the intensity of central areas of the electron radiation field for flattening of the beam profile. Each of the steel layers had $1 \mathrm{~mm}$ thickness. The simulated geometry for producing the mixed beam is illustrated in Figure 1.
Figure 1. The Used Geometry in the Monte Carlo Simulation for Producing the Mixed Beam, Placed Above of the Applicator

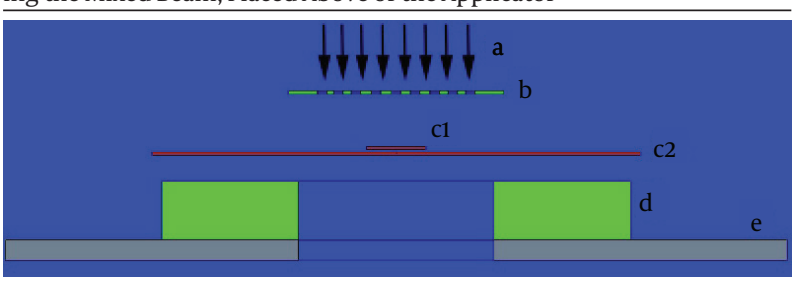

a) The exited electron beam from the head of linac collimator incidence on the b) perforated Lead target, c1) The first steel scattering foil for reducing the intensity of center of electron beam, c2) The second scattering foil d) The Lead shield for blocking the scattered photons and electrons, e) The applicator holder tray.

\section{Results}

\subsection{The Tuning of the Simulation}

The measured R50 and RP for $10 \times 10 \mathrm{~cm}^{2}$ field were 6.5 and $7.8 \mathrm{~cm}$, respectively, and these values in simulation were 6.6 and $7.9 \mathrm{~cm}$. Therefore the $\mathrm{E}_{0}$ (mean) in measurement and simulation are equal to 15.1 and $15.4 \mathrm{MeV}$. Other points of the PDD curve were had smaller than $2 \%$ difference between measurement and simulation. The penumbra $20 \%-80 \%$, the distance between $20 \%$ and $80 \%$ dose points of profile curve, at the depth of $1 \mathrm{~cm}$ for both of measurement and simulation were $1.2 \mathrm{~cm}$, which increased to 5.5 and $5.4 \mathrm{~cm}$ for measurement and simulation at the depth of $7 \mathrm{~cm}$. Due to the compliance of measure and simulated PDD and profiles, the mixed beam production components placed at the electron beam path.

\subsection{The Simulations With the Mixed Beam Produc- tion Layers}

By accounting the perforated Lead layer and steel scattering layers in the beam interactions, the mixed beam was produced. The PDD and profile of $10 \times 10 \mathrm{~cm}^{2}$ field were calculated for same depths same as the pure electron beam. The PDD of the mixed beam, has a shrink buildup region and a smoother dose fall-off after this part of PDD curve in comparison with the pure electron beam. The photon percentage for $1 \mathrm{~mm}$ thickness with $0.2,0.3$, and $0.5 \mathrm{~cm}$ holes diameter Lead layer target was about $33 \%, 32 \%$, and $28 \%$ and for $2 \mathrm{~mm}$ targets punched with $0.2,0.3$, and $0.5 \mathrm{~cm}$ holes, the $\mathrm{x}$-ray percentages were $43 \%, 41 \%$, and $35 \%$. Also, without the target layer the simulation is repeated and the contaminated photon was $15 \%$, that mostly produced by scattering layers. These percentages were measured at the depth of $R_{P}$, which is beyond of the practical range of electrons $\left(R_{P}\right)$.

The $\mathrm{R}_{100}, \mathrm{R}_{50}, \mathrm{R}_{\mathrm{P}}$ and $\mathrm{R}_{\mathrm{P}}$, are equal to 1.6, 5.5, 6.7 and $8.2 \mathrm{~cm}$ for $1 \mathrm{~mm}$ target with $0.3 \mathrm{~cm}$ holes. Also, the surface dose was reduced to $80 \%$ that was $92 \%$ for pure $16 \mathrm{MeV}$ electron and $49 \%$ for pure $6 \mathrm{MV}$ photon. The PDDs of produced mixed beam by 1 and $2 \mathrm{~mm}$ targets illustrated in Figure 2. Also, the different of electron, photon, and mixed beam PDDs is shown in Figure 3. 

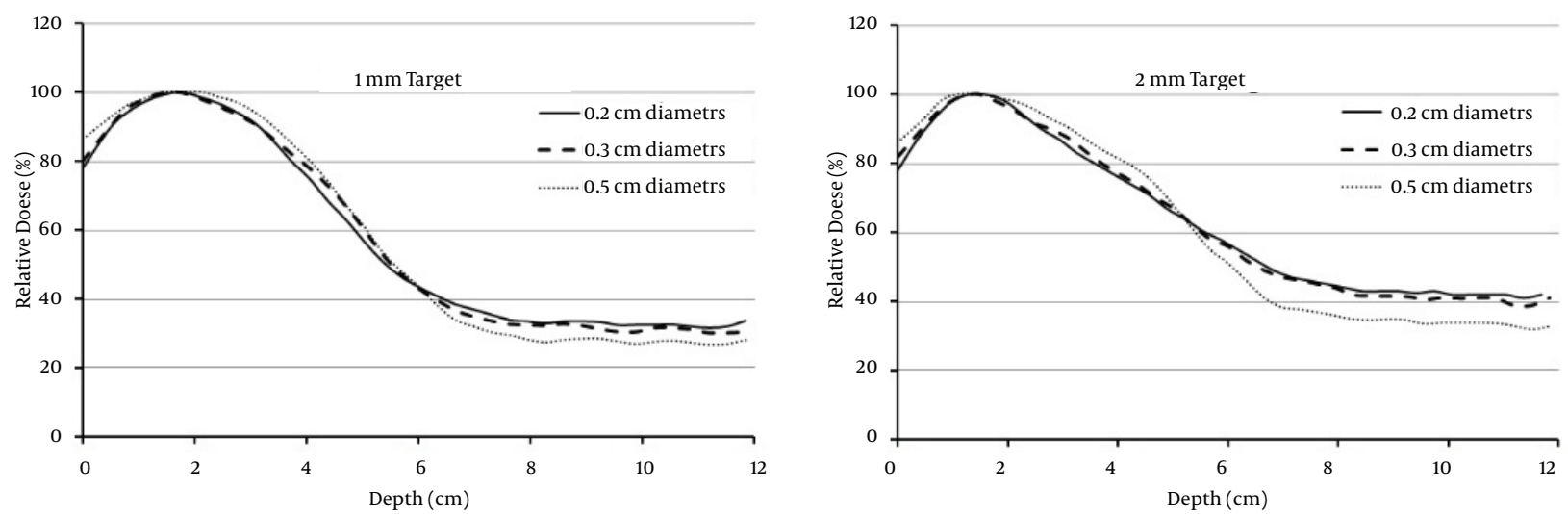

Figure 2. The PDDs of $10 \times 10 \mathrm{~cm}^{2}$ Field Size for the Mixed Beam Produced by 1 and $2 \mathrm{~mm}$ Lead Targets With Various Punches Diameters

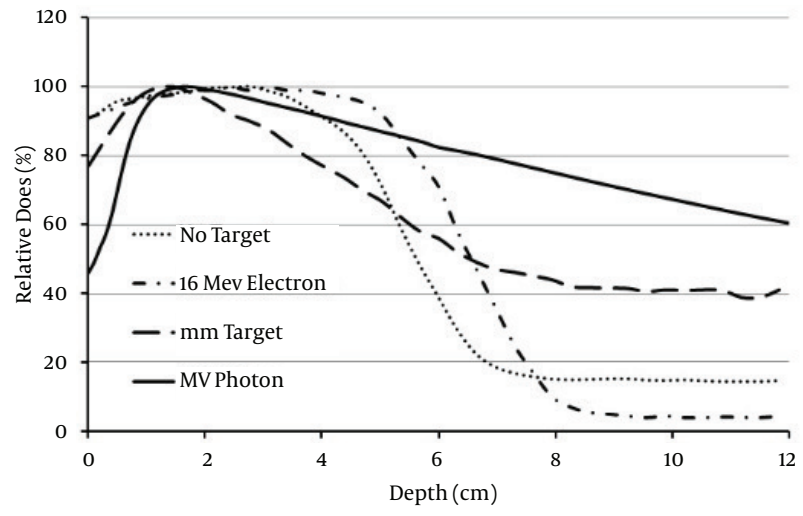

Figure 3. The Comparing of the PDDs for Different Beams

The penumbra, at the same depths of simulation, didn't showed a significant different between various targets with same thickness. For example, at the depth of $3.5 \mathrm{~cm}$ it was changed from 3.8 to $4.0 \mathrm{~cm}$ while the diameters of the target holes changed from 0.2 to $0.5 \mathrm{~cm}$ for $1 \mathrm{~mm}$ thickness target, and changed from 4.9 to $4.8 \mathrm{~cm}$ for 2 $\mathrm{mm}$ thickness target. Also, the penumbra in that depth without the target was $4.7 \mathrm{~cm}$.

Figure 4 shows the profile of pure $16 \mathrm{MeV}$ electron and the mixed beams. In this figure the difference of penumbra changing by depth is obvious. The percentage of penumbra changes with the depth is obviously smaller for mixed beam in comparison with the pure electron. The minimum variation of penumbra was observed for $2 \mathrm{~mm}$ target with $0.2 \mathrm{~cm}$ holes. In general, the $2 \mathrm{~mm}$ target had a smaller changes in penumbra with the depth.
In comparison with the pure electron, the mixed beam shows a useful specificity for treatment of semi-deep targets, which the dose field size is not suffering from the large changes. In the case of $2 \mathrm{~mm}$ target with 0.2 holes the penumbra variation is $21 \%$ which it is $31 \%$ for pure photon and $350 \%$ for pure electron beam.

\subsection{Small Field Size (Cut-Outs)}

\subsubsection{The $4 \times 4 \mathrm{~cm}^{2}$ Cut-Out}

The $\mathrm{R}_{50}, \mathrm{R}_{\mathrm{P}}$, and $\mathrm{R}_{\mathrm{p}}$, in the pure electron mode were 6.6, 7.9 , and $8.0 \mathrm{~cm}$ which by inserting the $4 \times 4 \mathrm{~cm}^{2}$ width cut-out they are reduced to $5.7,7.3$, and $7.6 \mathrm{~cm}$. The flatness at the depths of 1 and $7 \mathrm{~cm}$ were $101 \%$ to $120 \%$. Also, the penumbras changes were $80 \%$.

But for the mixed beam, produced by $1 \mathrm{~mm}$ target with $0.3 \mathrm{~cm}$ holes, without and with this cut-out the $\mathrm{R}_{50}$ were 5.5 and $5.4 \mathrm{~cm}$ and $\mathrm{R}_{\mathrm{p}}$, were 8.8 and $8.9 \mathrm{~cm}$. The changes of PDD parameters has a slight variation in comparison with the $10 \times 10 \mathrm{~cm}^{2}$ field size. Aside from these, the overall shape of the PDDs of mixed beam for cut-out mode and $10 \times 10 \mathrm{~cm}^{2}$ field size are very similar. Also, the flatness was $102 \pm 1 \%$ for the three depths. The penumbras changes between 1 and $7 \mathrm{~cm}$ depths were $-40 \%$ that shows dose fall-off sharpening for profile at the deeper depths. This is arise from the fact that the effect of wide target and geometrical penumbra is reduced by using the cut-out.

The penumbra and flatness changes were $-6 \%$ and $3 \%$ for $2 \mathrm{~mm}$ target. In comparison with the $1 \mathrm{~mm}$ target the penumbras changes of $2 \mathrm{~mm}$ target was smaller. Also, the PDD parameters were almost constant with a good approximation, relative to the $10 \times 10 \mathrm{~cm}^{2}$ field size. 

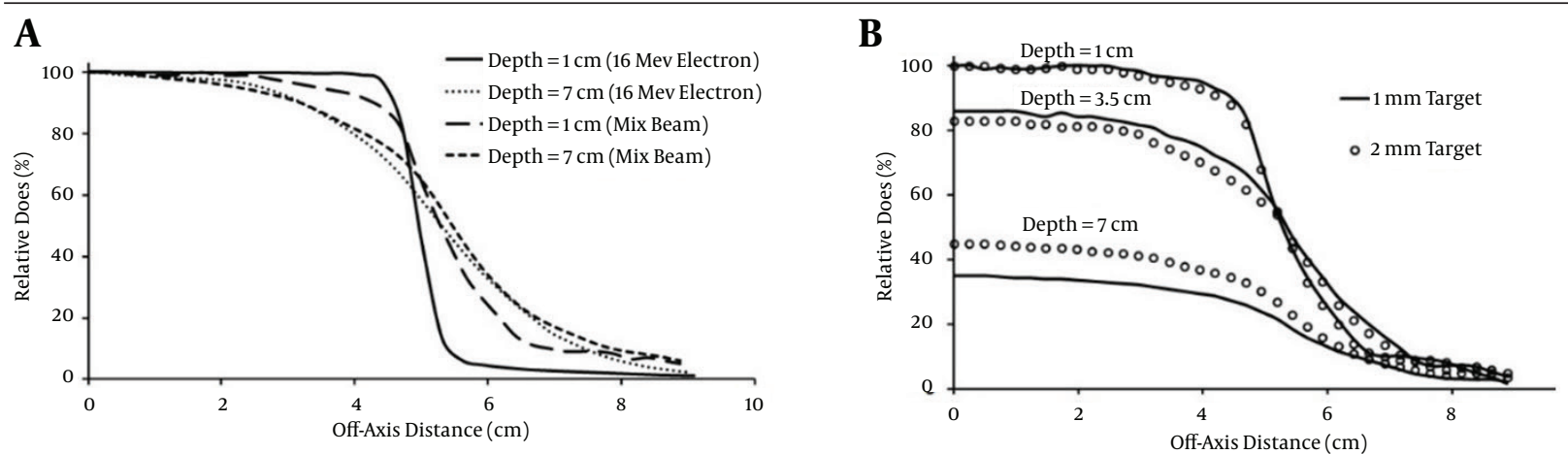

Figure 4. A, The Comparison of Beam Profiles of Pure $16 \mathrm{MeV}$ Electron and the Mixed Beam Produced by $1 \mathrm{~mm}$ Target Configuration, and B, Comparison of Beam Profile for and $2 \mathrm{~mm}$ Targets Configurations

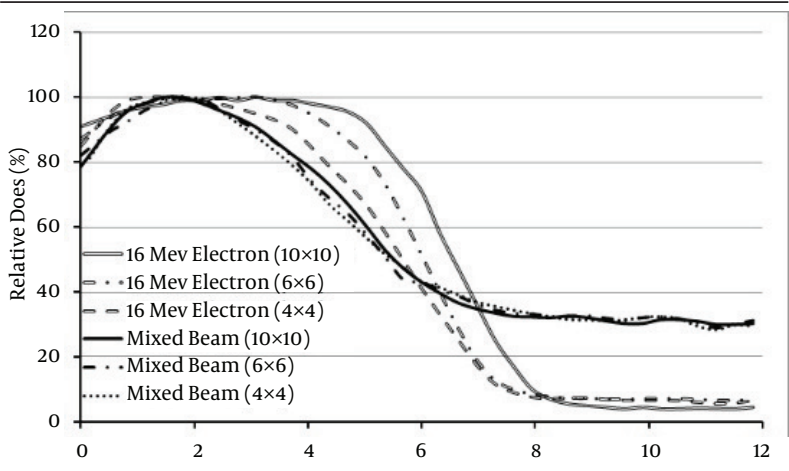

Figure 5. The PDD Curves of Mixed Beam (1 mm Target, $0.3 \mathrm{~cm}$ Punches Diameters) and $16 \mathrm{MeV}$ Electron Beam for $4 \times 4 \mathrm{~cm}^{2}, 6 \times 6 \mathrm{~cm}^{2}$, and $10 \times$ $10 \mathrm{~cm}^{2}$ Cut-Outs

\section{Discussion}

This study showed the feasibility of production and employment of the simultaneous mixed electron and photon beam, and its ability to improve some of the disadvantages of electron beam. On the other hand, the simultaneous mixed beam can acts as a new type of therapeutic beam due to its advantages over the electron or photon beam for specific cases. Also, by modifying the shape and configuration of the conventional linacs target and flattening filter the mixed beam would be produced by future types of linacs. Also, there is no need to remove or insert the add-ons like the electron applicator while shifting between electron and photon mode for intra-fractional beam mixing, in comparison with the conventional methods.

Also, the surface dose was between electron and photon beam. Therefore, when we need a beam with surface dose lesser than electron (or higher than photon) and/or a deep dose different than electron and photon beam, the mixed beam can act as an alternative beam. The $2 \mathrm{~mm}$ target showed a better capabilities in the most case of penumbra, cut-out, and PDD parameters changes, therefore, it may be more preferable to the $1 \mathrm{~mm}$ target. The PDDs of this new beam, indicated the new range of energy deposition depth and a significant stability in the depth dose parameters while using the cut-outs.

\section{References}

1. Hoppe BS, Stegman LD, Zelefsky MJ, Rosenzweig KE, Wolden SL, Patel SG, et al. Treatment of nasal cavity and paranasal sinus cancer with modern radiotherapy techniques in the postoperative setting-the MSKCC experience. Int J Radiat Oncol Biol Phys. 2007;67(3):691-702

2. Xiong W, Li J, Chen L, Price RA, Freedman G, Ding M, et al. Optimization of combined electron and photon beams for breast cancer Phys Med Biol. 2004;49(10):1973-89.

3. Khaledy N, Arbabi A, Sardari D. The effects of cutouts on output, mean energy and percentage depth dose of 12 and $14 \mathrm{MeV}$ electrons. J Med Phys. 2011;36(4):213-9.

4. Korevaar EW, Heijmen BJ, Woudstra E, Huizenga H, Brahme A. Mixing intensity modulated electron and photon beams: combining a steep dose fall-off at depth with sharp and depth-independent penumbras and flat beam profiles. Phys Med Biol.1999;44(9):2171-81.

5. Korevaar EW, van Vliet RJ, Woudstra E, Heijmen BJ, Huizenga $\mathrm{H}$. Sharpening the penumbra of high energy electron beams with low weight narrow photon beams. Radiother Oncol.1998;48(2):213-20.

6. Vatanen T, Traneus E, Lahtinen T. Dosimetric verification of a Monte Carlo electron beam model for an add-on eMLC. Phys Med Biol. 2008;53(2):391-404.

7. Khan FM. The Physics of Radiation Therapy. 5th ed. Lippincott Williams \& Wilkins; 2009.

8. Khaledi N, Arbabi A, Sardari D, Rabie Mahdavi S, Aslian H, Dabaghi $\mathrm{M}$, et al. Monte Carlo investigation of the effect of small cutouts on beam profile parameters of 12 and $14 \mathrm{MeV}$ electron beams. Radiat Meas. 2013;51-52:48-54.

9. Krayenbuehl J, Oertel S, Davis JB, Ciernik IF. Combined photon and electron three-dimensional conformal versus intensity-modulated radiotherapy with integrated boost for adjuvant treatment of malignant pleural mesothelioma after pleuropneumonectomy. Int J Radiat Oncol Biol Phys. 2007;69(5):1593-9.

10. Mosalaei H, Karnas S, Shah S, Van Doodewaard S, Foster T, Chen J The use of intensity-modulated radiation therapy photon beams for improving the dose uniformity of electron beams shaped with MLC. Med Dosim. 2012;37(1):76-83.

11. International Atomic Energy Agency. Absorbed Dose Determination in External Beam Radiotherapy: An International Code of Practice for Dosimetry based on Standards of Absorbed Dose to Water TRS I. 398. Vienna: International Atomic Energy Agency; 2000.

12. Pelowitz D. MCNPX User's Manual Report LA-CP-07-1473. Los Alamos National Laboratory; 2008.

13. Turian JV, Smith BD, Bernard DA, Griem KL, Chu JC. Monte Carlo calculations of output factors for clinically shaped electron fields.J Appl Clin Med Phys. 2004;5(2):42-63.

14. Cuttone G. Applications of particle accelerators in medical physics. Int J Radiat Oncol Biol Phys. 2008;72(6):193-9.

15. Shimozato T, Okudaira K, Fuse H, Tabushi K. Monte Carlo simulation and measurement of radiation leakage from applicators used in external electron radiotherapy. Phys Med. 2013;29(4):388-96. 